\title{
Strange Energy Loss and Chaotic Entropy of Domain-Wall Motion
}

\author{
Hikaru Okuno \\ Institute of Engineering Mechanics and Systems, University of Tsukuba, 1-1-1, Tennoudai, Tsukuba, Ibaraki 305-8573
}

The strange behavior of thermal energy loss in chaotic motion of a Bloch wall is studied by using entropy. The thermal energy loss due to domain-wall motion is calculated by integrating a damping term. The entropy of domain-wall motion is calculated by using the probability of the state-points distribution in the phase space. The results of computer simulation show that the thermal energy loss in the periodic window of the bifurcation diagram increases in spite of the decrease in randomness of domain-wall motion, which seems to conflict with the first and second laws of thermodynamics. The chaotic entropy $S_{c}$ of domain-wall motion is introduced into the equation of energy balance to solve the above conflict. $S_{c}$ is a kind of "mechanical entropy", and differs from thermodynamic entropy. The entropy $S$ of the system is expressed as the sum of the chaotic entropy $S_{c}$ of the domain-wall motion in the phase space and the entropy $S_{m}$ related to the thermal energy loss by using the property of additivity of entropy. The results of computer simulation are interpreted as indicating that the value of Temperature $\times \int_{1}^{2} d S_{m}$ (the thermal energy loss) increases so that it compensates for the decrease of Temperature $\times \int_{1}^{2} d S_{c}$ (the randomness of motion) in the periodic window. It is explained that the thermal energy loss increases in spite of the decrease in randomness of motion in the periodic window.

Key words: magnetic domain wall, magnetization processes, chaos, energy loss, entropy

\section{Introduction}

Chaos of the magnetic spin dynamics has been a very interesting problem in the basic magnetism. ${ }^{1-3} \mathrm{~A}$ model of the Bloch wall with a term for a nonlinear restoring force has been shown to undergo chaotic motion at a certain amplitude and frequency of magnetic drive fields. ${ }^{4,5}$ The study of chaotic domain-wall motion is very important to solve the problems of energy loss and recording errors or noise in the field of applied magnetism. ${ }^{4,5}$

We previously reported the strange behavior of energy loss when a domain-wall showed a chaotic motion and a regular motion alternately with increasing the magnetic drive field. ${ }^{4}$ The energy loss due to domain-wall motion in the periodic window increased in spite of decrease in the randomness of motion. Examples of the time series of the position and velocity of the domain-wall motion were presented to explain above behavior. The velocity of domain-wall motion in the periodic window was larger than that in the chaotic region and caused the increase of energy loss. The intrinsic reason why the energy loss due to domain-wall motion increased in the periodic window has not been made clear. The energy loss due to domain-wall motion with increasing the frequency of magnetic drive field also shows the very interesting behavior that the peak of energy loss shifts and collapses when the chaotic domain-wall motion happens. ${ }^{5}$

In this paper, the above strange behavior of energy loss is discussed from the point of view of thermodynamics by using the entropy of domain-wall motion.

\section{Model and calculation}

The dynamics of a Bloch wall motion are expressed by a nonlinear differential equation ${ }^{5}$ with the terms of inertia, damping, restoring, and a magnetic drive force by using the Landau-Lifshitz-Gilbert equation ${ }^{6-8}$ as follows:

$$
\begin{array}{r}
{\left[\frac{2 \mu_{0}\left(1+\alpha^{2}\right)}{\gamma^{2} \Delta}\right] \frac{d^{2} x}{d t^{2}}+\left[\frac{8 \pi \mu_{0} I_{s} \alpha}{|\gamma| \Delta}+\frac{16 d I_{s}{ }^{2}}{\pi^{3} \rho}\right] \frac{d x}{d t}} \\
+\left[2 I_{s} H_{c}\right] \sin \frac{2 \pi x}{\ell}=\left[2 I_{s} H\right] \cos \omega t,
\end{array}
$$

where $\mu_{0}$ is the permeability of vacuum, $\alpha$ is the Gilbert damping coefficient, $\gamma$ is the gyromagnetic constant, $\Delta$ is the width parameter of the magnetic domain wall, $x$ is the coordinate of the domain wall, $t$ is the time, $I_{s}$ is the saturation magnetization, $d$ is the thickness of the magnetic material, $\rho$ is the electrical resistivity of the magnetic material, $\delta=\pi \Delta$ is the width of the domain wall, $H_{c}$ is the coercive force, $\ell$ is the wavelength of the coercivity-inducing potential, $H$ is the external magnetic field, and $\omega=2 \pi f$ is the angular frequency of the external magnetic field. The coefficients in brackets are defined as $M, K, R$, and $B$ in that order.

An energy loss due to domain-wall motion $U$ is calculated by integrating the damping term as follows: ${ }^{4}$

$$
U=\int D \cdot d x=\int K\left|\frac{d x}{d t}\right| d x \quad\left(\mathrm{~J} / \mathrm{m}^{2}\right)
$$

where $D$ is the damping force.

The time series data of velocity are used as the values of $\mathrm{dx} / \mathrm{dt}$. The integral is performed in the range of 200 periods of the magnetic drive field. 
Table 1 Material parameters.

\begin{tabular}{lc}
\hline Thickness of film & $d=0.25 \mu \mathrm{m}$ \\
Resistivity & $\rho=160 \mu \Omega \mathrm{cm}$ \\
Domain-wall width & $\delta=0.1 \mu \mathrm{m}$ \\
Coercive force & $H_{c}=0.29 \mathrm{~A} / \mathrm{m}$ \\
Magnetization & $I_{s}=0.46 \mathrm{~T}$ \\
g factor & $\mathrm{g}=2$ \\
Wavelength of stress & $\ell=1 \mu \mathrm{m}$ \\
Relaxation frequency & $\nu=1 \times 10^{9} \mathrm{~s}^{-1}$ \\
\hline
\end{tabular}

The chaotic entropy $S_{c}$ of domain-wall motion is calculated by using the probability of the state-points distribution in the phase space as follows:

$$
S_{c}=-k_{B} \sum_{i=1}^{N} p_{i} \log _{e} p_{i},
$$

where $k_{\mathrm{B}}$ is the Boltzmann constant, $N=50$ is the total number of the volume element in the phase space and $p_{i}$ is the probability that the state points are in the i-th volume element. The phase space is one-dimensional with one potential wavelength $\ell=1 \mu \mathrm{m}(-\ell / 2 \sim \ell / 2)$, because the potential is periodic and the periodic boundary condition is used. The state points are obtained at every the same phase of the magnetic drive field.

The computer simulation is performed by the fourth-order Runge-Kutta method. The material parameters are shown in Table 1 . The values of coefficients, magnetic field, and the initial values are shown in Table 2.

\section{Results and discussion}

\subsection{Relation between bifurcation diagram, energy loss and entropy}

The Bloch wall motion and the thermal energy loss were calculated by increasing the coefficient $B$ of the external force that was proportional to a magnetic drive field with the radio frequency. The value of the energy loss shows an interesting behavior when it is compared with the bifurcation diagram.

Figure 1 shows the bifurcation diagram of domain-wall velocity versus amplitude of the external drive field at a frequency $f=2 \mathrm{MHz}^{4}$

Table 2 Values of the coefficients, magnetic field, and initial values.

$\begin{array}{lc}\text { Mass of domain wall } & \mathrm{M}=1.66 \times 10^{-9} \mathrm{~kg} / \mathrm{m}^{2} \\ \text { Damping coefficient } & \mathrm{K}=1.74 \times 10^{-2} \mathrm{~kg} / \mathrm{sm}^{2} \\ \text { Restoring coefficient } & \mathrm{R}=0.2668 \mathrm{~kg} / \mathrm{ms}^{2} \\ \text { Magnetic field amplitude } & H=0.217-0.467 \mathrm{~A} / \mathrm{m} \\ \text { Magnetic field frequency } & f=2 \mathrm{MHz} \\ \text { Initial values } & x=\mathrm{d} x / \mathrm{d} t=0\end{array}$

Figure 2 shows the energy loss of the domain-wall motion in the same range of $B$ in Fig. $1 .^{4}$ Figure 3 shows the entropy of the domain-wall motion in the same range of $B$ in Fig. 1.

The magnetic domain-wall motion changes from the regular motion to the chaotic motion as shown in Fig. 1 when the magnetic domain wall crosses over the tops of several potential wells at about $B=0.25$. The chaotic motion returns to the periodic motion and the periodic window appears at about $B=0.29$ and $B=0.36$. The periodic point splits to two points at $B=0.389$, four points at $B=0.397$ and the doubling continues. The route to chaos above $\mathrm{B}=0.36$ is the period-doubling bifurcation. ${ }^{4}$

The energy loss of the magnetic domain-wall motion increases on a large scale as shown in Fig. 2 when the domain-wall motion goes into the chaos for the first time at $B=0.25$. The entropy of domain-wall motion $S_{c}$ increases at the same time as shown in Fig. 3, which is calculated by using the probability of the state-points distribution in the phase space. It is consistent with our intuition that the energy loss increases with increasing the randomness of motion.

The appearance of the energy loss and the entropy of domain-wall motion in the periodic window ( at $\mathrm{B}=0.36$ $\sim 0.39$ ) are different from the above behavior and the change of each value is opposite. The domain-wall motion is periodic. Nevertheless, the energy loss in the periodic window is larger than that of the chaotic motion in the neighborhood. The entropy of domain-wall motion $S_{c}$ in the periodic window is zero. The energy loss increases in spite of the decrease in randomness of domain-wall motion. This seems to conflict with the first and second laws of thermodynamics.

\subsection{Internal energy of system and input energy to system}

The following discussion is made to explain the above problem. The point is to consider both the complexity, namely, mechanical entropy in a chaotic motion and the thermodynamic entropy.

The system A consists of a ferromagnetic material and a magnetic domain wall in that. The energy valance of the system $\mathrm{A}$ is given by

$$
d E=d W
$$

where $E$ is the internal energy of the system $\mathrm{A}$ and $W$ is the energy input to the system A.

The equation (4) can be written in the isothermal process as follows:

$$
d E=d F+T d S
$$

where $F$ is the free energy, $T$ is the absolute temperature, and $S$ is the entropy of the system A.

The system A has many spins, but only spins in the 


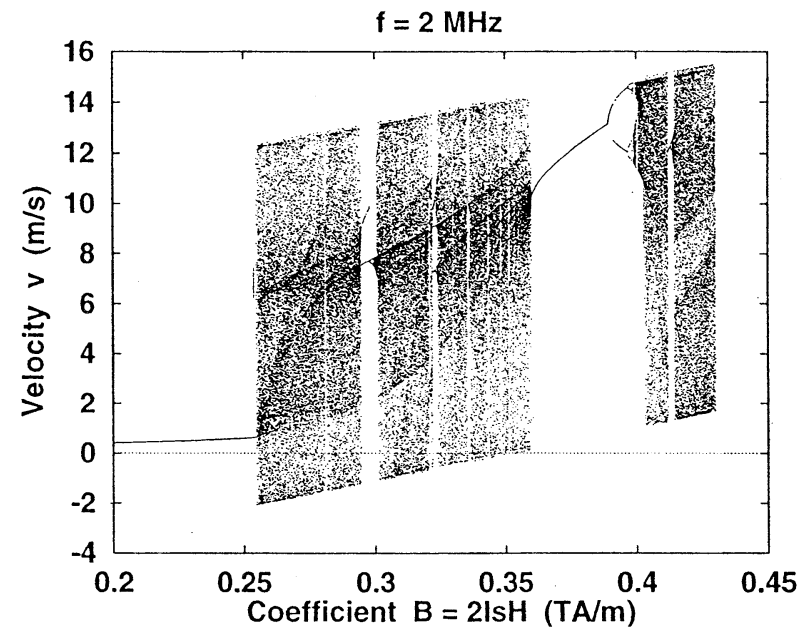

Fig.1 Bifurcation diagram of domain-wall velocity versus amplitude of the external drive field at frequency $f=2 \mathrm{MHz}$.

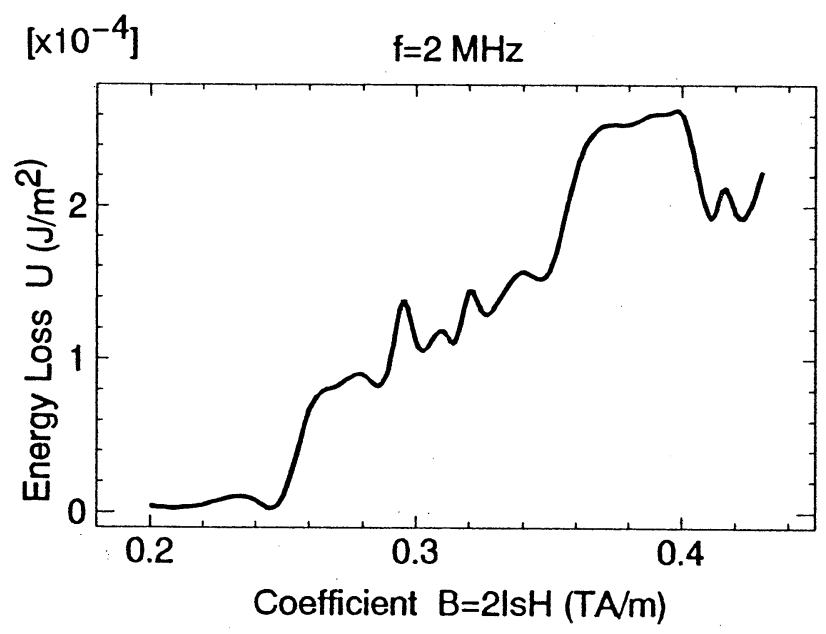

Fig.2 Energy loss due to domain-wall motion in the same range of $B$ in Fig. 1 .

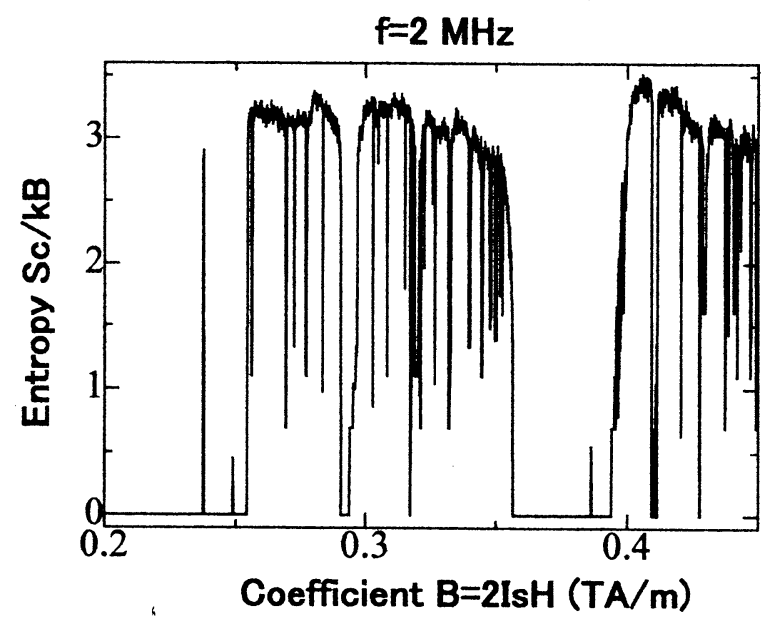

Fig.3 Entropy of domain-wall motion in the same range of $B$ in Fig. 1. domain wall are rotated by the magnetic drive field and the rotation propagates in the magnetic material with the material parameters in Table I and $f=2 \mathrm{MHz}$. Therefore, the system $\mathrm{A}$ is simplified as a macroscopic model that one domain wall with the force of inertia, damping, restoring moves in a drive field. The energy input to $A$ is used for the domain-wall motion and the energy dissipation. Consequently, it is considered that the free energy of this system consists of a kinetic energy $Q$ and a potential energy $P$ of a domain wall. The equation (5) can be written as follows:

$$
d E=d Q+d P+T d S .
$$

If the entropy $S$ of the system A consists of the chaotic entropy $S_{c}$ and the thermodynamical entropy $S_{m}, d S$ is written by using the property of additivity of entropy as follows:

$$
d S=d S_{c}+d S_{m},
$$

where $S_{c}$ is the entropy which shows the complexity of the macroscopic and chaotic motion of a magnetic domain wall, and $S_{m}$ is the entropy which shows the energy dissipation to the microscopic motion in a magnetic material.

Equation (4) is written by using the equations (6) and (7) as follows:

$$
d E=d Q+d P+T d S_{c}+T d S_{m}=d W .
$$

When the system changed from the state 1 to the state 2 , the equation (8) is written as follows:

$$
\int^{2} d E=\int^{2} d Q+\int^{2} d P+T \int^{2} d S_{c}+T \int^{2} d S_{m}=\int^{2} d W,
$$

where $T \int_{1}^{2} d S_{m}$ corresponds to the energy loss $U$ of the domain-wall in the equation (2).

\subsection{Thermal energy loss and entropy at the first chaos-in}

Consider the case that the domain-wall motion goes into the chaos for the first time at about $B=0.25$ with increasing the energy input, namely, a magnetic drive field with the radio frequency.

Figure 4 shows the magnetic drive force and the time series of the magnetic domain-wall position at $B=0.22$ and $B=0.27$. Figure 5 shows the time series of the domain-wall velocity at the same conditions.

The domain-wall motion is regular within one potential well $(-\ell / 2 \leq x \leq+\ell / 2)$ below $B=0.25$. The domain wall crosses over the tops of several potential wells immediately above $B=0.25$ and the mode shifts from the regular motion to the chaotic motion as shown in Fig. 4 and Fig.5.

The restoring force for the domain wall is $R \sin (2 \pi x / \ell)$ and the potential is obtained by $-\int[(R / M) \sin (2 \pi x / \ell)] d x=-(\ell R / 2 \pi M) \cos (2 \pi x / \ell)$.

The external drive force is $B \cos \omega t$. The direction of 


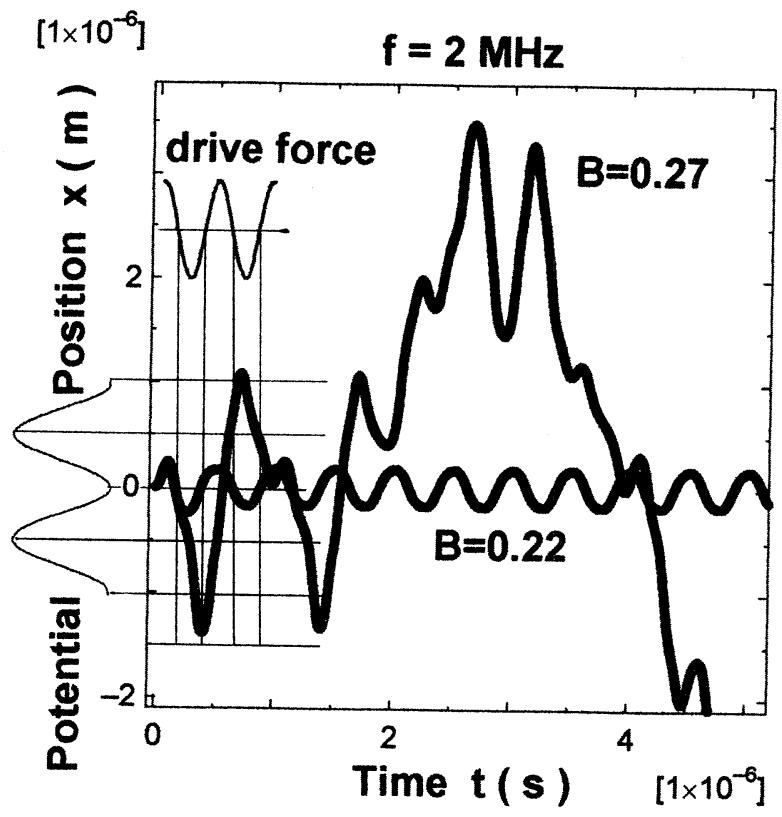

Fig.4 Time series of domain-wall position at $\mathrm{B}=0.22$ ( periodic motion within one potential), $\mathrm{B}=0.27$ ( chaotic motion over several potentials ) and external drive force.

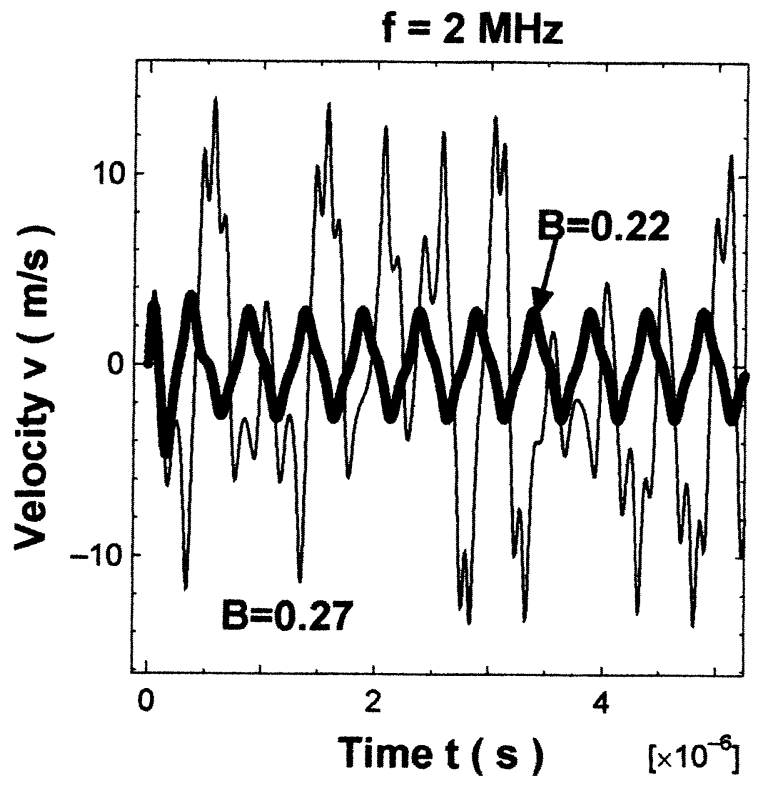

Fig.5 Time series of domain-wall velocity at $\mathrm{B}=0.22$ and $\mathrm{B}=0.27$.

restoring force of domain wall by the potential is always toward the equilibrium point $x=0$ and opposite to the external drive force in the region $-\ell / 2 \leq x \leq+\ell / 2$ immediately below $B=0.25$ as shown in Fig.4. The input energy by the external drive force is consumed as the potential energy by the restoring force. In this case, the sign of $\int_{1}^{2} d P$ is plus, because the sign of $\int_{1}^{2} d W$ is plus in the equation (9) of the energy valance.

On the other hand, there is the duration that the restoring force of domain wall is in the direction toward the equilibrium point $x= \pm n \ell, n=1,2,3 \cdots$ and accelerates the domain wall in the same direction of the external drive force in the region $-n \ell \leq x \leq-n \ell / 2$ and $+n \ell / 2 \leq x \leq+n \ell$ immediately above $B=0.25$. The rectangular areas as shown in Fig. 4 ( from $0.125 \sim 0.375$ $\mu \mathrm{s}$ and $-0.5 \sim-1 \mu \mathrm{m}$, from $0.375 \sim 0.625 \mu \mathrm{s}$ and $+0.5 \sim+1 \mu \mathrm{m})$ are the duration of acceleration. These areas periodically exist. The sign of $\int d P$ in the energy valance equation (9) is minus in that duration.

Consequently, the equation of the potential energy immediately above $B=0.25$ compared with that immediately below $B=0.25$ is as follows:

$\int_{1}^{2} d P \quad($ at $B=0.25-\delta)>\int_{1}^{2} d P \quad$ (at $B=0.25+\delta$ ),

where $\delta$ shows the small amount of $B$.

The energy input $\int_{1}^{2} d W$ immediately above $B=0.25$ is almost the same as that immediately below $B=0.25$, because $\delta$ is small. The equation is as follows:

$$
\begin{aligned}
& \int^{2} d Q+\int^{2} d P+T \int^{2} d S_{c}+T \int^{2} d S_{m}(\text { at } B=0.25-\delta) \\
& \cong \int^{2} d Q+\int^{2} d P+T \int^{2} d S_{c}+T \int^{2} d S_{m} \quad(\text { at } B=0.25+\delta) .
\end{aligned}
$$

From the equation (10) and (11), the next equation is obtained as follows:

$$
\begin{aligned}
& \int^{2} d Q+T \int^{2} d S_{c}+T \int^{2} d S_{m}(\text { at } B=0.25-\delta) \\
& <\int^{2} d Q+T \int^{2} d S_{c}+T \int^{2} d S_{m} \quad(\text { at } B=0.25+\delta) .
\end{aligned}
$$

This is consistent with the increases of both the energy loss $T \int_{1}^{2} d S_{m}$ and the entropy $\int_{1}^{2} d S_{c}$ at $B=0.25$ as shown in Fig. 2 and Fig. 3 respectively.

\subsection{Thermal energy loss and entropy in periodic window}

The energy loss in the periodic window immediately below $B=0.4$ is compared with that of the chaotic motion immediately above $B=0.4$.

Figure 6 shows the time series of the magnetic domain-wall position at $B=0.38$ and $B=0.41$. The domain-wall motion at $\mathrm{B}=\mathbf{0 . 3 8}$ is periodic and the motion at $B=0.41$ is chaotic. Figure 7 shows the time series of the domain-wall velocity at $\mathrm{B}=0.38$.

Figure 8 shows the time series of the domain-wall velocity at $B=0.41$. The amplitude of the velocity at $\mathrm{B}=0.38$ is almost the same as that at $\mathrm{B}=0.41$.

The energy input $\int_{1}^{2} d W$ immediately above $B=0.4$ is almost the same as that immediately below $B=0.4$, because $\delta$ is small. The equation is obtained as follows:

$$
\begin{aligned}
& \int^{2} d Q+\int^{2} d P+T \int^{2} d S_{c}+T \int^{2} d S_{m}(\text { at } B=0.4-\delta) \\
& \cong \int^{2} d Q+\int^{2} d P+T \int^{2} d S_{c}+T \int^{2} d S_{m}(\text { at } B=0.4+\delta)
\end{aligned}
$$




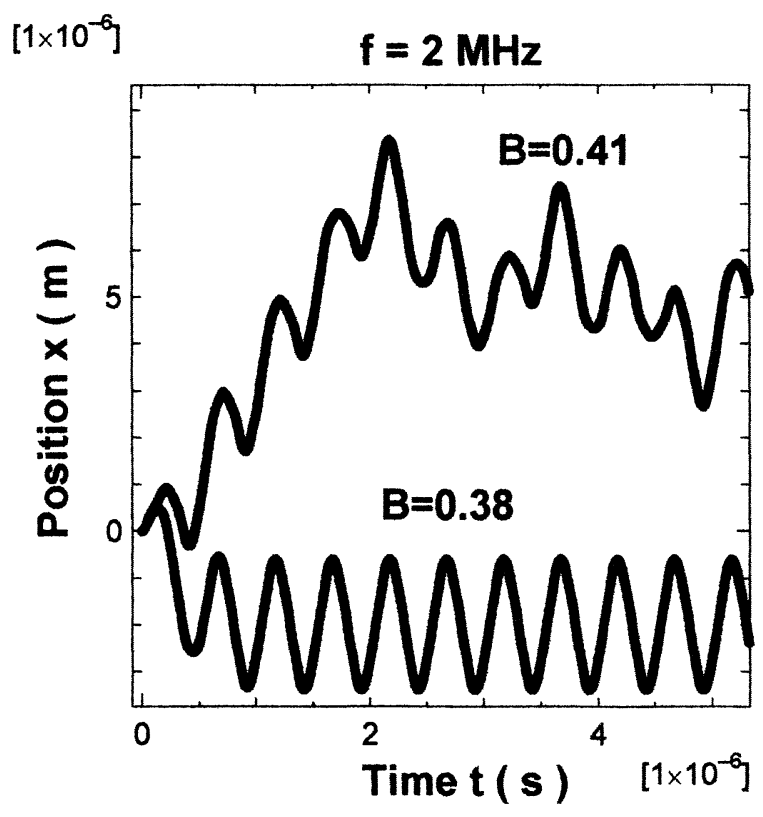

Fig.6 Time series of domain-wall position at $\mathrm{B}=0.38$ ( periodic window) and $\mathrm{B}=0.41$ ( chaotic region).

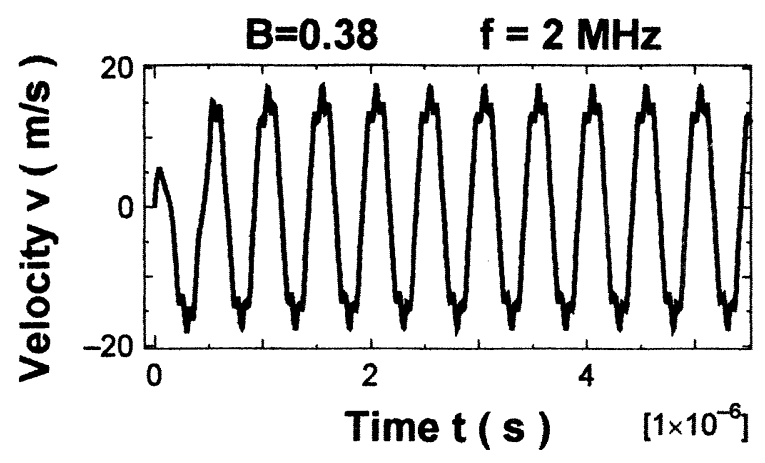

Fig.7 Time series of domain-wall velocity at $\mathrm{B}=0.38$.

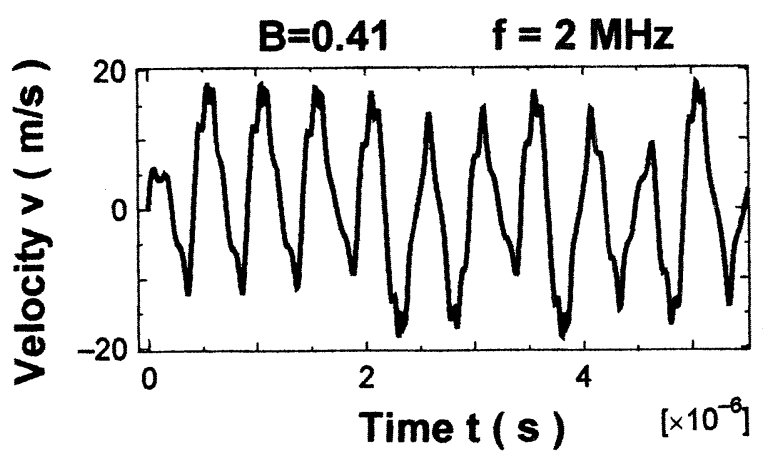

Fig.8 Time series of domain-wall velocity at $B=0.41$.
Both domain-wall motions at $\mathrm{B}=0.38$ and at $\mathrm{B}=0.41$ have the same mode in which the domain wall crosses over the tops of several potentials. The duration of minus $\int d P$ happens at $\mathrm{B}=0.38$ as well as at $\mathrm{B}=0.41$ in the same frequency. The value of $\int d P$ at $\mathrm{B}=0.38$, therefore, is almost the same as that at $\mathrm{B}=0.41$.

The value of $\int d Q$ at $\mathrm{B}=0.38$ is almost the same as that at $B=0.41$, because the amplitude of the velocity in Fig. 7 is almost the same as that in Fig.8.

Consequently, it is considered from equation (13) that the value of $T \int_{1}^{2} d S_{m}$ (at $B=0.4-\delta$ ) in the periodic window is larger than the value of $T \int_{1}^{2} d S_{m}$ (at $B=0.4+\delta$ ) in the neighbor chaos region so that $T \int_{1}^{2} d S_{m}$ compensates for the decrease of $T \int_{1}^{2} d S_{c}$,because $\int_{1}^{2} d S_{c}>0 \quad$ (at $\left.\mathrm{B}=0.4+\delta\right)$ and $T \int_{1}^{2} d S_{c}=0 \quad($ at $B=0.4-\delta)$.

This term of $T \int_{1}^{2} d S_{m}$ is a dissipative energy, namely, an energy loss. Equation (13) is consistent with that an energy loss increases in spite of the decrease in randomness of motion at a periodic window in Fig. 2 .

\section{Conclusion}

The domain-wall motion in the periodic window is regular. Nevertheless, the energy loss is larger than that of the chaotic motion in the neighborhood by the computer simulation. The entropy of domain-wall motion in the periodic window is zero. The energy loss increases in spite of the decrease in randomness of domain-wall motion.

To explain the above phenomenon, the entropy $S$ of the system $\mathrm{A}$ is expressed as the sum of the chaotic entropy $S_{c}$ of the domain-wall motion in the phase space and the entropy $S_{m}$ related to the energy loss by using the property of additivity of entropy.

The energy input $\int_{1}^{2} d W$ immediately above $B=0.4$ ( chaos region )is almost the same as that at immediately below $B=0.4$ ( regular region ). Therefore, it is possible that the value of $T \int_{1}^{2} d S_{m}$ (the energy loss) in the periodic window is larger than the value in the chaotic region, because $T \int_{1}^{2} d S_{c}$ (the randomness of motion) in the periodic window is 0 . It is explained that an energy loss increases in spite of the decrease in randomness of motion in the periodic window.

\section{Acknowledgments}

The author wishes to thank the Japanese Ministry of Education, Science, Sports and Culture for a Grant-in-Aid for Scientific Research (C).

\section{References}

1) P.E. Wigen, H. Dötsch, Y. Ming, L. Baselgia and F. Waldner: J. Appl. Phys. 63, 4157 (1988).

2) D.W. Peterman, M.Ye and P.E. Wigen: J. Appl. Phys. 76, 
6886 (1994).

3) V.S. Gornakov, V.I. Nikitenko, I.A. Prudnikov and V.T. Synogach: Phys. Rev. B46, 10829 (1992).

4) H. Okuno, Y. Sugitani and T. Homma: IEEE Trans. Magn., 30, 4305 (1994).

5) H. Okuno: J. Appl. Phys., 81, 5233 (1997).

6) L. Landau and E. Lifschitz:Phys. A Sov. Union, 8, 153(1935).

7) T. L. Gilbert: Phys. Rev., 100, 1234(1955).

8) J. C. Slonczewski: Int. J. Mag., 2, 85(1972).

(Received June 26, 2001; Accepted October 26, 2001) 